\title{
Effect of Surface Spinal Stimulation (SSS) on H-reflex in Normal Individuals
}

\author{
Narkeesh', A., Navroop kaur², N. \& Sharma ${ }^{3}$, S.
}

${ }^{\mathbf{1}}$ Associate Professor, Email: narkeesh@ gmail.com, ${ }^{2}$ \& 3 Post Graduate Students,

Department of Physiotherapy, Punjabi University, Patiala-147002, Punjab, India

\section{Abstract}

Surface spinal stimulation is a well known technique that uses electrical currents in order to activate nerves and innervating muscles, it is one of the primary tools used to restore functions in various neurological disorders by reducing spasticity. There are many previous studies where surface spinal stimulation has been used in various neurological disorders and purposes but still there is no study to explain how surface spinal stimulation influences on monosynaptic reflex. The present study has used $2500 \mathrm{~Hz}$ as a carrying frequency and $20 \mathrm{~Hz}$ as a beat frequency. Electrodes are attached $5 \mathrm{~cm}$ apart at the para spinal area at the T10-L2 level and electrode size $(9 \times 4.5 \mathrm{~cm})$. The present study applied medium frequency currents to produce only sensory stimulation and was applied for duration of 45 minutes. $\mathrm{H}$ reflexes are taken pre and post spinal stimulation measure the soles muscle. The mean, standard deviation and $\mathrm{t}$ value for all the variables were calculated and was concluded that spinal stimulation had a significant effect on monosynaptic reflex as there was significant decrease in $\mathrm{H} / \mathrm{M}$ ratio and $\mathrm{H}$ amplitude, however, there elongation of $\mathrm{H}$ latency but not up to significant levels was observed.

Key words: H-Reflex, Surface Spinal Stimulation

\section{Introduction:}

Electrical Stimulation is a technique that uses electrical currents to activate nerves innervating muscles and is primarily used to restore functions in people with disabilities. Surface Spinal Stimulation (SSS) is a form of electrical stimulation, and is used to influence the activity of nerve root fibers under the Para vertebral muscle through the surface electrodes applied over the skin of Para vertebral muscles (Wang et al, 2000). SSS is the process of stimulating the spinal cord with various electrical currents in order to produce stimulation and reduction in spasticity at the corresponding level of body segment. Spinal cord electrical stimulation is used in cerebral palsy and multiple sclerosis in reducing spasticity in the majority of subjects along with improvement in bladder function, respiratory function, volitional control; active and passive movement and mood state with carry over effects lasting from 30 minutes to 24 hours (Hazelwood et al, 1994).

The H-reflex (Hoffmann reflex) is a reflectory reaction of muscles after electrical stimulation of sensory fibers (Ia afferents stemming from muscle spindles) in their innervating nerves (for example, those located behind the knee). The $\mathrm{H}$ reflex test is performed using an electric stimulator, which gives usually a squarewave current of short duration and small amplitude (higher stimulations might involve alpha fibers, causing an F-wave, compromising the results), and an EMG set, to record the muscle response. That 
response is usually a clear wave, called $\mathrm{H}$ wave, 28-35 ms after the stimulus, not to be confused with an F-wave. An M-wave, an early response, occurs 3-6 ms after the onset of stimulation. The $\mathrm{H}$ and $\mathrm{F}$-waves are later responses. As the stimulus increases, the amplitude of the F-wave increases only slightly, and the H-wave decreases, and at supramaximal stimulus, the $\mathrm{H}$-wave will disappear. The $\mathrm{M}$-wave does the opposite of the H-wave. As the stimulus increases the $\mathrm{M}$-wave increases. There is a point of minimal stimulus where the M-wave is absent and the $\mathrm{H}$ wave is maximal. The present study put on efforts to explore the effects of surface spinal stimulation in 25 normal adolescents and to know how it influences the monosynaptic reflex.

\section{Materials and Methods}

Study was performed on 25 subjects taken from the Punjabi University, Patiala under the age group of 20-25 years. It was an experimental study which was performed in the Department of Physiotherapy. Study was performed in accordance with ethical considerations of the institute and their consent was taken prior to the study. Before beginning with the procedure, the subjects who were selected on the basis of convenient sampling by applying inclusion criteria and were explained the entire procedure in detail. The inclusion criterion was Male subjects of age 20 to 25 years, with normal BMI between 19 to $25 \mathrm{~kg} / \mathrm{m}^{2}$ and with height $165-190 \mathrm{~cm}$ are included. The body temperature ranges between 36-37 ${ }^{\circ} \mathrm{C}$, leg length from $27-49 \mathrm{~cm}$ and absence of any systemic, physical or neurological problem. The Exclusion Criteria was any sensory or motor impairment, space occupying lesion, upper motor neuron or lower motor neuron symptoms, mixed symptom rigidity, flaccidity and spasticity, any neurological and cognitive deficit, any radiculopathy and neuropathy, any recent surgery on back, hip, knee, height below $165 \mathrm{~cm}$ or above $190 \mathrm{~cm}$, leg length less than $27 \mathrm{~cm}$ or more than 49 $\mathrm{cm}$ and subjects with BMI more than 25 or less than 19. Of all the 25 subjects, Hreflex was recorded maintaining room temperature between $19-24^{\circ} \mathrm{C}$ on nondominant side in prone lying. Recording and reference electrodes were placed at the soleus muscle and sub maximal stimulation was given at popliteal crease. Surface Spinal Stimulation was delivered by pulses that were generated from amplitude modulated alternating current of a carrier frequency of $2500 \mathrm{~Hz}$, and modulated to deliver beat at a frequency of $20 \mathrm{~Hz}$. For application, an electrode was placed on each side of the spine $(5 \mathrm{~cm}$ apart) over the paravertebral skin at the twelfth thoracic and first lumbar vertebral levels. The self-adhesive electrodes of rectangular shape and size of $5 \times 9 \mathrm{~cm}$ were used. The stimulation amplitude was adjusted for each subject to produce only sensory stimulation and it was applied continuously for 45 minutes (Wang et al., 2000).

Surface spinal stimulation was then applied at T12 \& L1 level for 45 minutes in prone lying and again H-reflex was recorded in prone lying. Pre and post stimulation values of latency of H-reflex (ms), H/M ratio and mplitude of $\mathrm{H}$-wave $(\mathrm{mV})$ were taken. The data was collected and analyzed.

\section{Results}

Statistics application was performed by using SPSS 16. Results were calculated by using 0.05 level of significance. Table 1 shows the mean and standard deviation of age. The mean and 
standard deviation of age is $22.36 \pm 1.57$ years.

Table 1: Mean and SD of Age

\begin{tabular}{ccc}
\hline Demographic & Mean & SD \\
\hline AGE & 22.36 & 1.57 \\
\hline
\end{tabular}

Further, it was found that glycated haemoglobin (HbAlc) was positively and significantly related with total cholesterol $(\mathrm{r}=0.29)$, high density lipoproteins $(\mathrm{r}=0.19)$, triglycerides $(\mathrm{r}=0.26)$ and very low density lipoproteins $(\mathrm{r}=0.16)$. However, low density lipoproteins $(r=0.5)$ did not show any significant relationship with $\mathrm{HbA1c}$.

Table 2. Mean and SD of H-Latency, $\mathrm{H} / \mathrm{M}$ and $\mathrm{H}$ amplitude at Pre and Post interval for the subjects included in the study

\begin{tabular}{ccccc}
\hline Variable & \multicolumn{2}{c}{ Pre } & \multicolumn{2}{c}{ Post } \\
& \multicolumn{2}{c}{ Stimulation } & \multicolumn{2}{c}{ Stimulation } \\
\cline { 2 - 5 } & Mean & SD & Mean & SD \\
\hline H-Latency & 28.46 & 0.93 & 28.10 & 1.22 \\
H/M & 1.76 & 0.77 & 1.10 & 0.65 \\
H Amplitude & 4.60 & 2.07 & 4.05 & 1.87 \\
\hline
\end{tabular}

Table 2 describes the mean and standard deviation of H-latency, H/M and $\mathrm{H}$ amplitude at Pre and Post interval. The mean and standard deviation of H-latency pre- stimulation is $28.46 \pm 0.93$ and post stimulation is $28.10 \pm 1.22, \mathrm{H} / \mathrm{M}$ is $1.76 \pm 0.77$ and $1.10 \pm 0.65$ and of $\mathrm{H}$ amplitude is $4.60 \pm 2.07$ and $4.05 \pm 1.87$.

Table 3: Comparison of mean value for H-Latency between Pre and Post interval for the subjects included in the study

\begin{tabular}{ccc}
\hline Variable & \multicolumn{2}{c}{$\begin{array}{c}\text { Pre Stimulation Vs Post } \\
\text { Stimulation }\end{array}$} \\
\cline { 2 - 3 } H-Latency & t value & P value \\
H/M & 1.982 & P $>0.05$ \\
H Amplitude & 6.567 & P $<0.05$ \\
& 2.279 & $P<0.05$ \\
\hline
\end{tabular}

Table 3 shows the comparison of mean value for H-latency between pre and post Interval for subjects included in the study. The $t$ value for H-latency pre vs. Post Stimulation is 1.982 at $\mathrm{P}>0.05$, $\mathrm{t}$ value for $\mathrm{H} / \mathrm{M}$ pre vs. post is 6.567 at $\mathrm{P}<0.05$ and for $\mathrm{H}$ amplitude pre vs. post is 2.279 at $\mathrm{P}<0.05$.

\section{Discussion}

Surface spinal stimulation is important intervention measure used in various neurological disorders like stroke for reducing spasticity (Walker, 1982). The possible physiological mechanism of tone reduction could be by the indirect activation of golgi tendon organ through electrically elicited contraction, there is also reported evidence that low threshold afferent input can change the activity in interneurons and alpha motor neurons by segmental propriospinal or supraspinal pathways (Visser and Zilvold, 1978). Surface spinal stimulation has pronounced effects in $\mathrm{H}$ reflex, which is an electrically induced reflex analogues to mechanically induced spinal stretch reflex, its arc is similar to spinal stretch reflex except that it bypasses the muscle spindle, and therefore it is a valuable tool for assessing mono synaptic reflex activity in spinal cord. Thus, it is reliable tool for assessment of muscle tone through the excitability of alpha motor neuron (Palmieri et al, 2004).

In the present study, to find the effect the effect of surface spinal stimulation in H-reflex, only male subjects were taken because the significant role of sex in H-reflex has already been elicited (Huang et al, 2009). H-reflex has also shown modifications due to variations in age and leg length 
(Robinson et al, 1988). So in present study, considering the age factor, subjects of age group 20-25 years and leg length in present study was controlled between 27 and $49 \mathrm{~cm}$. H - reflex latency shows increase with cooling and decrease with warming (Riccardo et al, 2001). So, in present study room temperature was monitored from $19-24^{\circ} \mathrm{C}$. As Body Mass Index has also shown to influence the conduction changes (Buschbacher, 1999), the subjects with normal BMI (19-25 $\mathrm{Kg} / \mathrm{m}^{2}$ ) were included in the study.

In the present study, surface spinal stimulation of carrier frequency of $2500 \mathrm{~Hz}$ and beat frequency $20 \mathrm{~Hz}$ was given through the surface electrodes of size $4.5 \times 9 \mathrm{~cm}$, which were placed $5 \mathrm{~cm}$ apart on each side of spine, as explained by Wang et al (2000). In order to find the changes in $\mathrm{H}$-latency, $\mathrm{H} / \mathrm{M}$ ratio and $\mathrm{H}$-amplitude on applying the Surface Spinal Stimulation an experimental was conducted on 25 subjects. Surface Spinal Stimulation was applied for 45 minutes over $\mathrm{T}_{12}$ and $\mathrm{L}_{1}$ Para vertebral skin and then pre and post stimulation readings of $\mathrm{H}$-latency, $\mathrm{H} / \mathrm{M}$ ratio and $\mathrm{H}$-amplitude were collected and analyzed through SPSS 16. The results revealed that there are changes in the nerve excitability, $\mathrm{H}$ amplitude and $\mathrm{H} / \mathrm{m}$ ratio and there is significant influence of Surface Spinal Stimulation on monosynaptic reflex there is significant decrease in $\mathrm{H} / \mathrm{M}$ ratio ( $\mathrm{t}$ value : 6.67) and $H$ amplitude ( $t$ value : 2.279), however there is elongation of $\mathrm{H}$ latency but not up to significant level.

Though the difference in the pre and post values was not statistically significant but there was considerable effect of spinal stimulation on H-latency. There has been less literature available to explain the mechanism behind the changes in value of H-latency after surface spinal stimulation. A decrease in H-latency value Post stimulation is explained by Wang et al., (2000), he stated that repetitive sensory stimulation causes sensory habituation due to generalized desensitization of spinal pathways, thus, it results in suppression of interneuron and hence the results were non-significant.

The $\mathrm{t}$-value for $\mathrm{H} / \mathrm{M}$ was 6.567 at $\mathrm{P}<0.05$ which was significant. These results supported by earlier studies, that there were significant changes in pre and post stimulation amplitude and $\mathrm{H} / \mathrm{M}$ values. The possible mechanism involved in reduction of the mean of $\mathrm{H} / \mathrm{M}$ ratio is that the spinal electrical stimulation induces lasting changes in $I_{a}$ fibre motoneuron synapse, thus spinal stimulation is valuable measure in modulating spinal motor pathways.

The t-value for $\mathrm{H}$-amplitude was 2.279 at $\mathrm{P}<0.05$ which was significant. The results are in accordance with the studies conducted by Hayashi et al, (1992) concluded that there is suppression of the H-reflex amplitude after the application of electrical stimulation. Gruber et al, (2007) also investigated that there is reduction in peak to peak amplitude of stretch reflex and $\mathrm{H} / \mathrm{M}$ ratio after the application of electrical stimulation for 30 minutes over the surface of muscle.

The study also considered certain limitations; the study was done only on normal males on narrow age group 20-25 years. The sample size was small. Skin resistance was not uniformly controlled, only application of spirit to reduce the skin resistance was done. These limitations have made a scope for future study in the same area. The future scope is Study can be done on large sample size and both sexes. Study can be done on 
individuals with tonal abnormalities along with various neurological disorders. Wide range of frequencies and duration of Surface Spinal Stimulation can be taken to find various effects in monosynaptic reflex.

\section{Conclusion}

The present study concluded that surface spinal stimulation has significant influence over the monosynaptic stretch reflex. The present study found that surface spinal stimulation of medium frequency current with a beat frequency of $20 \mathrm{~Hz}$ had an inhibitory influence over the monosynaptic stretch reflex and it also significantly reduced $\mathrm{H} / \mathrm{M}$ ratio and $\mathrm{H}$ amplitude along with prolonged $\mathrm{H}$ latency but not up to the mark of significance.

\section{References}

Buschbacher, R. 1999. Normal range for H-reflex recording from the calf muscle S., Am. $J$. Phys. Med. And Rehab., $77: 6$.

Gruber M, Taube W, Gollhofer A, Beck S, Amtage F, Schubert M. 2007. Training-specific adaptions of $\mathrm{H}$ - and stretch reflexes in human soleus muscle. J. Mot. Behav., 39: 68-78.

HaHyashi, R., Tako, Kenji., Tokuda, Takahiko. and Yanagisawa, Nobuo. 1992. "Comparison of amplitude of human soleus H-reflex during sitting and standing", Neuroscience research, 13(3): pp 227-233.
Hazelwood, M. E., Brown, J. K., Rowe, P. J. 1994. The use of therapeutic electrical stimulation in the treatment of hemiplegic cerebral palsy. Dev. Med. Chil. Neurol., 36: 661-673.

Huang, C.R., Chang, W.N., Chang, H.W., Tsai, N.W., and LU, C. 2009. Effects of Age, Gender, Height and Weight on late responses and nerve conduction study parameters. Acta. Neurol. Taiwan, 18: 242-249.

Palmieri, R.M., Ingersoll, C.D. and Hoffman, M.A. 2004. The Hofmann Reflex: Methodologic considerations and applications for use in sports medicine and athletic training research", J. Ath. Train., 39(3): 268-77.

Riccardo, M., Giovani, B.S., Aido, M.,Vitaliano, F.M., Lucio, P. 2001. Recruitment curve of the Soleus H-reflex in chronic back pain and lumbosacral radiculopathy. BMC Muscle Skeletal Disorders, 4: 11.

Robinson, C., Kett, N., Bolam, J. 1988. Spasticity in Spinal Cord injured patients: 2. Initial measures and long term effects of surface electrical stimulation. Arch. Phys. Med. Rehabil., 69: 862-8.

Visser, S.L., Zilvold, G. 1978. EMG analysis in hemiplegic patients treated by means of FES of the Peroneal nerve. Scand. J. Rehab. Med., 10: 75-9.

Walker, J.B. 1982. Modulation of Spasticity : Prolonged Suppression of a Spinal reflex by electrical stimulation. Science, 216: 203-4.

Wang, R.Y., Chan, R.C., Tsai, M.W. 2000. Effect of thoraco-lumbas electric sensory stimulation on knee extensor spasticity of persons who survived cerebrovascular accident (CVA). J. Rehabil. Res. Dev., 37(1): 73-9. 